\title{
Digitalization in Healthcare and Telecommunication Support Systems in Medicine
}

\author{
Kukhtevich I.I.* \\ Penza State Institute of Advanced Medical Education, \\ Penza, Russia \\ e-mail: gvv17@ya.ru
}

\author{
Goryunova V.V. \\ Penza State University \\ Penza, Russia \\ e-mail: gvv3317@ya.ru
}

\author{
Goryunova T.I. \\ V.A. Trapeznikov Institute of Control Sciences of Russian \\ Academy of Sciences \\ Moscow, Russia \\ e-mail: tatjanagoryunova20@gmail.com
}

\author{
Zhilyaev P.S. \\ Penza State University \\ Penza, Russia \\ e-mail: itmmbs17@mail.ru
}

\begin{abstract}
Digital transformation and information integration provide operational management of information and communication processes in many industries. The use of digital and telemedicine technologies in healthcare is particularly important. The article presents the directions of development of telecommunication projects in medicine and considers the main tools and applications for creating an automated medical history in a digital environment. The methods of intellectual support of complex test solutions for the electronic medical history in the field of teleneurology are described. The methods of knowledge representation in telemedicine systems using rule-templates are considered. It is noted that the rule base can accelerate the comparison of patient data and the development of diagnostic hypotheses, providing data search and their distribution into separate groups in accordance with the context of a particular medical problem. The authors note that the stage of conceptual analysis or structuring of knowledge is traditionally (along with the extraction stage) a "bottleneck" in the life cycle of developing intelligent decision support systems. The development of intellectual support systems for integrated test solutions in the field of neurology belongs to this class of complex systems, since they have the main signs of complexity (hierarchy of concepts, intra-element and inter-element communication, etc.). The complexity of designing such systems is determined mainly by the complexity of subject areas, as well as the complexity of describing the behavior of individual subsystems.
\end{abstract}

Keywords - digital technology, healthcare, telemedicine, teleneurology, knowledge representation, decision making

\section{INTRODUCTION}

Information systems based on the digitalization of not only economy, but also society as a whole, become an integrator of breakthrough technologies when enterprises turn into digital platforms integrated into the modern development of society [1]. This approach, supported by the ideology of the digital economy, is the most progressive trend, primarily for healthcare enterprises [2].

The digital economy in healthcare is developing in accordance with the basic principles of systems theory [3], A mandatory process of improvement and transformation is required, aimed at qualitative changes and their reflection in the models of information communications, primarily in telemedicine systems (TMIS). In the world of modern digital technologies, telemedicine is inseparable from the digitization of all processes associated with caring for a person's condition and his health. In the era of the digital economy, doctors need to interact with huge arrays of reference information and use intelligent information support to organize systematic monitoring of patient data and decision-making in real time.

Digitalization and the active use of cloud and intelligent information technologies in modern healthcare require access to telemedicine systems for a wide range of medical specialists at all stages of diagnosis and treatment [4]. Automated medical history allows you to represent important components of the processes of diagnosis and treatment in the form of a choice of compact rule templates.

The authors note the possibility of using ontological support tools to integrate decision making [5].

Particular attention is paid to the development of diagnostic processes and the presentation of knowledge for complex test solutions in the field of teleneurology.

\section{DIGITALIZATION IN HEALTHCARE AND TELECOMMUNICATION PROJECTS IN MEDICINE}

Digitalization of healthcare primarily provides for the creation of a global telecommunications network in medicine, the idea of which is being promoted by the World Health Organization. There are more than 250 telemedicine projects in the world, most of which are clinical [6]. In addition, telemedicine has developed educational, informational and analytical projects [7].

There are platform solutions for the effective implementation of telemedicine. In particular, the american project "Doctor on Demand" is a platform that allows doctors to conduct round-the-clock meetings with patients through video communications. The peculiarity of the system is that doctors can not only diagnose the disease, but also write an electronic prescription for the medicine. The average patient 
waiting time for a doctor is 4 minutes, $97 \%$ of all patients expressed satisfaction with the services provided, and the cost of the service is $4-5$ times lower than when visiting the doctor personally.

Telemedicine kiosks are becoming an important part of US medical programs. We can ask for help at such a kiosk directly from the workplace, since the employer pays for the consultation as part of social insurance programs [8]. According to analysts, the American market for such stalls from 10 thousand "seats" in 2015 will grow to at least 36 thousand in 2020. The market of insurance services is actively developing using information insurance kiosks.

The British East Midlands Ambulance Service (EMAS) was able to save $£ 40$ million and eliminate 116,000 unnecessary calls in a year thanks to Advanced Health \& Care Odyssey Clinical Support.

Due to telemedicine, the number of hospitalizations in the Netherlands decreased by 64 percent, outpatient visits by 39 percent, and in the United States by 19 and 70 percent, respectively.

The development of international medical telecommunication networks is set for different purposes. For example, the Satellite system is used to disseminate medical knowledge in developing countries and train personnel. "Planet Heres" is a system of global scientific telecommunications, international scientific expertise and coordination of scientific programs, etc.

The largest telemedicine service provider in China plans to launch a network of " techno-doctor" mini-clinics the size of a telephone box that will be equipped with artificial intelligence. Such a "techno-doctor" will analyze patient complaints and send them to a specialist doctor who will diagnose and write recommendations.

\section{USE OF ELECTRONIC MEDICAL HISTORY AND THE METHODOLOGY OF INTELLECTUAL SUPPORT FOR SPECIALIST DECISIONS}

Automated medical history allows representing important components of the electronic medical history in the form of a selection of compact and more visible documents. Typically, specific classes of patient data are selected for this, such as active allergies, active problems, active treatment, and recent examinations. In this case, possible explanations of the observed anomalies can be dynamically provided.

The presence of medical records in a computer database does not exclude the work of finding the necessary fact, but allows the user to receive data in a clearly structured form (for example, in the form of reports on the progress of the disease), which facilitates the extraction of necessary information. More importantly, some TMIS are involved in the data retrieval process and can provide data related to a particular patient problem using rule bases. The rule base can accelerate the comparison of patient data and the development of diagnostic hypotheses, providing data search and distributing them into separate groups in accordance with the context of a particular medical problem.
Negotiable documents are computer-issued forms in which certain information is given and additional questions are asked. They are the paper equivalents of screen forms issued to video terminals. The forms of registration of visits, as well as super accounts are examples of negotiable documents .

Telemedicine systems can use two main ways of processing text to generate such narrative reports:

1. The layout of the phrases. This method is often used in systems of departments of radiation diagnostics, radiology and radiology to draw conclusions on normal results and on typical pathologies identified. It can also be used to formulate narrative conclusions that interpret the testimony of medical measuring devices, such as spirometer and electroencephalographs, as well as to form conclusions on surgical pathologies.

2. Filling in the templates. Many word processors offer a means of creating templates for standard letters or documents into which individual deviations can then be inserted. The template text consists of constant and variable components; to obtain the final document, the user fills in the variable part of the template with the data of a specific patient. An example is reception coupons; Variable components of the coupon are the name of the patient and his address, the date and time of admission, as well as the doctor who must receive the patient.

The structure of the telemedical electronic medical history can also be represented by several components:

- Short epicrisis (patient or teleconsultation identifier, gender, age, diagnosis, brief medical history, date of request, urgency of consultation) - conceptual structure Sk (text format for the implementation of 18 elements).

- Dynamic part the functional structure Sf, which contains a set of information (on the implementation of 42 elements).

- Questions for the consultant (“diagnosis?", "Treatment tactics?", “Assessment of treatment?", Etc.) - text format.

- Visualizing data (radiographs, clinical photographs, MRI, computer tomograms) - image files.

- Explanatory data (text accompanying the visualizing data: anatomical region, projection, method of coloring and contrasting, magnification, date of investigation, etc.) - text format.

- Text data (full extract from the medical history, test results, etc.) - text format.

Additional data (explanatory drawings, extract from the medical history, features of the medical diagnostic process, etc.) is an arbitrary (usually text) format.

\section{POSSIBILITIES OF INTELLECTUAL SUPPORT IN TELENEUROLOGY}

Teleneurology is part of telemedicine that offers counseling on neurological problems from a remote location using the telephone or the Internet. It includes teleconsultations, teleconferences and can be initiated by a 
doctor or patient. Some diseases that neurologists diagnose and treat include cognitive impairment, dementia, strokes, multiple sclerosis, and epilepsy.

Clinicians' initiative in using teleneurology is especially important in patients with stroke. Thrombolytic procedures should be performed urgently. Neurologists can now evaluate the patient using this technology, wherever he is. And the treatment of strokes depends on determining the nature of the stroke using MRI, assessing the severity of the stroke, the timing of the onset of the stroke, and the somatic status of the patient.

The treatment of epilepsy depends on the type of epileptic symptoms, their frequency and the age of a patient.

Using the knowledge base, TMIS can become so reasonable that it will independently choose the types of data the doctors need from the context of the medical history.

Automated medical history systems in telemedicine offer additional means for the formation of specialized forms, for example, tests to determine cognitive functions:

- SAGE Testing;

- MMSE test;

- Mini-Cog Test;

- GP-COG Clinical Dementia Test;

- BOMC Concentration Test;

- SLUMS test;

- Montreal MoCA Test;

- MIS memory rating;

- Short tests, etc.

The assessment of cognitive functions is an important task in many diseases of the nervous system, in particular, in diseases of the brain. Testing for cognitive impairment is also important for determining treatment tactics, evaluating the effect of ongoing therapy, and for solving many other tasks. To assess cognitive impairment, there are a large number of scales; one of the most popular is the MMSE scale. The name of the test was derived from the abbreviation - mini-mental state examination, in translation a mini-study of cognitive state. The test consists of several questions-tasks:

Definition of orientation (task $\boldsymbol{f l}$ ). The patient is asked what date is now (year, time of year, day, month, day of the week) for each correct answer, 1 point is added to the patient. Then they ask in which country, which city, in which district of the city, in which institution, on which floor is the patient, for each correct answer, a score is also added. In this section, therefore, the maximum possible number of points is 10 .

The definition of perception (task $\boldsymbol{f 2}$ ). The patient is asked to listen and repeat three words that are not related to each other (for example, an orange-chair-coin or an airplanewindow-rose). At the same time, he is warned that they will need to be reproduced in a few minutes. For each correctly repeated word add 1 point.
Determination of attention and ability to count (task $\mathbf{f 3}$ ). The patient is asked to orally subtract the number 8 from 200. Then 5 times, in a row (200-192-184-176-168-160). For each correct subtraction, one point is added. If the patient makes a mistake, one can ask whether he is sure of the answer.

The definition of memory functions (task $\mathbf{f 4}$ ). The patient is asked to recall the three words indicated in the second part. For each word -1 point.

The definition of the functions of speech, reading, writing (task f5).The patient is shown two objects (clock, pencil, neurological hammer, etc.). For each correctly named answer, 1 point is awarded.

The final task is drawing (task f6). They are asked to draw two intersecting pentagons. In this case, the completed task is considered correct if the intersection of two figures forms a quadrangle and all the corners of the pentagons are preserved. 1 point is also given.

From the above mentioned aspects, we can suggest the use of the corresponding components of the knowledge field $\boldsymbol{P z}$

$$
P_{z}=(I, O, M),
$$

where $\boldsymbol{I}$ is the structure of the source data to be processed and interpreted in the knowledge base; $\boldsymbol{O}$ - structure of the output data, that is, the result of the system; $\boldsymbol{M}$ - operational model of the subject area of testing, on the basis of which there is a modification of $\boldsymbol{I}$ in $\boldsymbol{O}$.

The inclusion of components $\boldsymbol{I}$ and $\boldsymbol{O}$ in $\boldsymbol{P z}$ is due to the fact that the components and structure of these interface components are implicitly (i.e. implicitly) present in the model of representation in the expert's task.

The operational model $\boldsymbol{M}$ can be represented as a combination of the conceptual structure $\boldsymbol{S k}$, which reflects the conceptual structure of the diagnostic subject area (for example, cognitive functions), and the functional structure $\boldsymbol{S f}$, which models the testing scheme for an expert doctor (composition of the test used):

$$
M=(S k, S f),
$$

where $\boldsymbol{S k}$ acts as a static, unchanging component of $\boldsymbol{P z}$, while $S f$ represents a dynamic, variable component.

$\boldsymbol{S} \boldsymbol{k}$ formation is based on the identification of the conceptual structure of the subject area and describes a fairly universal algorithm for conducting conceptual analysis based on a modification of the structural analysis paradigm and the construction of a hierarchy of concepts (the so-called "knowledge pyramid").

The structure $\boldsymbol{S f}$ includes the concepts of the diagnostic domain $\boldsymbol{A}$ and models the basic functional relationships $\boldsymbol{R} \boldsymbol{A}$ (or relations between the concepts) that form $\boldsymbol{S k}$. These relationships reflect the model or decision-making strategy in the selected subject area of diagnostics. Thus, $\boldsymbol{S} \boldsymbol{f}$ forms a strategic component of $\boldsymbol{M}$.

As a result of the analysis of the research methodology and description of the subject areas, information flows were 
analyzed, and the methodology for the study and description of the subject areas of diagnosis was determined.

As a result of the analysis, an analytical-multiple model of the TMIS subject area was obtained, in which the following elements were highlighted: automated functions; data processing tasks; automation objects; many types of TMIS users; information flows.

\section{DEVELOPMENT OF TOOLS FOR KNOWLEDGE REPRESENTATION AND DECISION MAKING USING TEMPLATES.}

The separation of the stages of extracting and structuring knowledge is very conditional, since a good knowledge engineer, already extracting knowledge, begins work on structuring and forming a field of knowledge.

However, at present, there is a tendency to be ahead of technological means of developing intelligent systems (ontology servers, expert systems, linguistic processors, training systems, etc.) with respect to their theoretical justification [10].

On the one hand, this is explained by the fact that from the first steps, the science of artificial intelligence (AI) was aimed at modeling poorly formalized semantic problems in which the traditional mathematical apparatus is not applicable; on the other hand, artificial intelligence is a branch of computer science and is actively developing as an industrial software industry [11].

The need to develop the theoretical foundations of science about methods for developing knowledge-based systems knowledge engineering - is substantiated in the works of D.A. Pospelova, E.V. Popova, V.L. Stefanyuk, R. Shenk, M. Minsky - leading experts in the field of AI in Russia and abroad [12].

The stage of conceptual analysis or structuring of knowledge is traditionally (along with the extraction stage) a "bottleneck" in the life cycle of developing intelligent systems [13]. The structuring methodology is close to the modern theory of large systems or complex systems, where traditionally the emphasis is on the design process of such systems. A great contribution to this theory was made by the classics of object-oriented analysis.

The development of intelligent TMIS can be reliably attributed to this class of tasks, since they have the main signs of complexity (a hierarchy of concepts, intra-element and inter-element communications, etc.). The complexity of designing TMIS is determined mainly by the complexity of subject areas and the management of the development process, as well as the complexity of ensuring the flexibility of the final software product and the description of the behavior of individual subsystems.

The design of complex systems and the methods of structuring information have traditionally used a hierarchical approach as a methodological technique for dividing a formally described system into levels (or blocks, or modules). At the highest levels of the hierarchy, the least detailed representations are used, reflecting only the most general features and features of the designed system. At the following levels, the degree of detail increases, while the system is not considered as a whole, but as separate blocks.

At each level, their ideas about the system and elements are introduced. The element of the $\boldsymbol{k}$-th level is a system for the level $\boldsymbol{k}-\boldsymbol{1}$, Advancement from level to level has a strict focus, determined by the design strategy - from top to bottom or from bottom to top.

The authors propose using an ontologically structured approach that allows combining two types of design strategies [14]. Descending or deductive STRtd (sequential decomposition of objects and processes from top to bottom, and up and inductive STRbu (from bottom to top), with a gradual generalization of concepts and increasing the degree of abstractness of descriptions from bottom to top.

The synthesis of these strategies, as well as the inclusion of the possibility of iterative returns to the previous levels of generalizations, made it possible to create a dual concept that provides an expert doctor with a wide palette of possibilities at the stage of structuring knowledge both for the formation of the conceptual structure of the diagnostic diagnostics subject area $\boldsymbol{S k}$ and for the functional structure $\boldsymbol{S} \boldsymbol{f}$.

The reason for stopping aggregation and disaggregation is the full use of the glossary of terms used by the expert, and the number of levels is a significant factor in the success of structuring.

Existing approaches to the design of complex systems can be divided into two large classes:

- A structural (system) approach or analysis based on the idea of algorithmic decomposition, where each module of the system performs one of the most important stages of the overall process.

- The object approach associated with the decomposition and allocation of not processes, but objects, with each object being considered as an instance of a particular class.

Structural analysis developed a large number of expressive tools for design, including graphical: data flow diagrams (DFD), structured dictionaries (thesauruses), specification systems, decision tables, arrow entity-relationship diagrams (ERDs), transition (state) diagrams, goal trees, flowcharts of algorithms (in the notation of Nassi - Schneiderman, Hamilton - Zeldin, Festl, etc.), tools project management (PERT diagrams, Gantt charts, etc.), environment models [15].

The multiplicity of tools and their certain redundancy are explained by the fact that each subject area, using the structural approach as a universal means of modeling, introduced its own terminology, which is most suitable for reflecting the specifics of a particular problem. Since knowledge engineering deals with a wide class of software (these are "soft" software), the task arises of developing a fairly universal structuring language.

The object-oriented approach (OOP), which emerged as a programming technology for large software products, is based on the following basic elementary concepts: objects, classes as 
objects connected by a common structure and properties, and classification as a means of streamlining knowledge; hierarchies with property inheritance; in-capsulation as a means of restricting access; methods and polymorphism for determining functions and relationships.

OOP has its own system of symbols and offers a rich set of logical and physical models for designing systems of high degree of complexity, while these systems are well structured, which makes it easy to modify them. The principle of OOP was first established in 1979, and then developed in.

The wide distribution of object-oriented programming languages $\mathrm{C}++, \mathrm{CLOS}$, Smalltalk, etc. successfully demonstrates the viability and promise of this approach.

It is possible to propose as a basic paradigm of the methodology of the structural analysis of knowledge and the formation of the knowledge field $\mathrm{Pz}$ a generalized objectstructured approach (OSP), consistently developed from mathematical justification to technology and software implementation .

The basic tenets of this paradigm are borrowed from the OSP and expanded.

1. Systematic (the relationship between the concepts).

2. Abstraction (identification of the essential characteristics of a concept that distinguishes it from others).

3. Hierarchy (ranking on ordered systems of abstractions).

4. Typing (allocation of concept classes with partial inheritance of properties in subclasses).

5. Modularity (dividing a task into sub-tasks or "possible worlds").

\section{Visibility and ease of notation.}

Using the fifth OSP postulate in knowledge engineering, it is possible to build global knowledge bases with the ability to isolate local problems using horizontal and vertical sections into separate modules of the space-description of the subject area.

The sixth postulate is listed last, but not by importance. In knowledge engineering, the formation of $\boldsymbol{P z}$ is traditionally a critical point, since the created informal model of the subject area should be extremely clear and concise. Traditionally, the language of knowledge engineering has been diagrams, tables, and other graphic elements that contribute to the visibility of representations. That is why the approach to the language proposed in this work is associated with a possible visualization of the design process.

A new methodology is considered in the work, which allows carrying out the structuring stage independently of the subsequent software implementation, relying on achievements in the field of developing complex systems, supporting the concept of ontologically structured approach (OSB).

The template-production method proposed by the authors consists in the fact that the relationships between the elements of the subject area, that is, between the sets, can be represented in the form of production rules, we will use logical variables as the conditions of the kernels, and when representing the actions, we apply unary operator functions implemented in the form patterns and logical expressions. For example, and $\boldsymbol{k}(\mathbf{1}$, $2,3,4,5)$ is a unary operation that assigns the value of the output variable o, taking into account the correct 5 responses of the patient. And the production rule "IF and $k(1,2,3)$ THEN f2: = 3" checks the correctness of the unary operation and assigns the total value $f 2:=3$ to the task $f 2$

A function template allows defining a family of functions. This family is characterized by a general algorithm that can be applied to data of various types. In this case, the specification of a specific data type for the next version of the function is provided by a special syntactic design called a list of parameters of the function template. A function declaration preceded by a list of template parameters is called a function template.

The syntax for declaring a template is determined by the following set of Backus - Naur sentences:

\section{Announcement: $=$ Template Announcement}

Template Declaration: = template <Template Parameter List> Announcement

Pattern Parameter List: = Pattern Parameter

:: = Pattern Parameter List, Pattern Parameter

Pattern Parameter: = Typical Parameter

$::=* * * * *$

\section{Type Parameter : = class Identifier}

The following is an example of a description of production rules for six typical tasks for determining cognitive functions. For the cognitive diagnostic domain model, these correspondences are presented as follows:

\section{IF f1 THEN and $k(1,2,3,4,5,6,7,8,9,10), M:=k$ \\ IF $f 2$ THEN and $k(1,2,3), M:=M+k$ \\ IF $f 3$ THEN and $k(1,2,3,4,5) M:=M+k$ \\ IF 44 THEN and $k(1,2,3), M:=M+k$ \\ IF f5 THEN and $\mathrm{k}(1,2,3,4,5), \mathrm{M}:=\mathrm{M}+\mathrm{k}$ \\ IF f6 THEN and $\mathrm{k}(1,2,3), \mathrm{M}:=\mathrm{M}+\mathrm{k}$}

A system analysis technique of TMIS has been developed, which allows creating the same type of system of various subject areas of medical diagnostics, which further ensures their integration into a single system. This technique includes the selection of many structural elements, the construction of the semantic adjacency matrix and the semantic reachability matrix, the selection of informational structures of groups, the selection of the sets of precedence and reachability for each structural element.

The use of ontologically oriented technologies complements the set of basic requirements regarding the integration of various subject areas of medical diagnostics using various mechanisms (APIs, web services, file sharing, etc.). It improves search capabilities through the use of search form templates and the use of complex search queries. In the 
integration model, the rule system works with a semantic network, the composition of the concepts and relations of which is determined by the ontology [16]. These capabilities can be used to implement methods for assessing and making managerial decisions of situational problems [17].

The use of the ontological knowledge base and modular ontologies is designed to provide intellectual support for realtime diagnostic and therapeutic processes.

\section{CONCLUSION}

The use of intellectual decision support and a knowledge base can be expanded through various mechanisms (web services, data exchange, etc.) in terms of integration with external systems, for example, an environmental health assessment system [18]

The proposed method for supporting complex test solutions improves the ability to monitor and diagnose patient conditions through the use of form templates and the use of complex search queries. In the integration model, the rule base works with a semantic network, the composition of the concepts and relations of which is determined by the ontology.

The introduction of the ontological knowledge base and modular ontologies is designed to provide intellectual support for real-time diagnostic and therapeutic processes.

The tools considered in the article allow determining classification groups based on the specified attributes and take into account the objective properties contained in the knowledge representation model. Such a model fully allows multi-valued data structuring, that is, correlation of one object with several classification groups, which may be necessary in connection with different approaches to data structuring for various functional tests.

The development of telemedicine systems is fully consistent with the general strategy of innovative development of Russia [19]

\section{References}

[1] V. Burkov, M. Goubko, N. Korgin, D. Novikov, Introduction to Theory of Control in Organizations. New York: CRC Press, 2015, 352 p.

[2] E. Brynjolfsson, B. Kahin, Introduction, Understanding Digital Economy. Cambridge: MIT Press, 2000, pp. 1-10.

[3] D. Novikov, A. Chkhartishvili, Reflexion and Control: Mathematical Models. London: CRC Press, 2014, 298 p.
[4] W. Froelich et al., "Case study: An example of the success of international telemedicine", J. of Telemedic. and Telecare, vol. 15, no. 4 pp. 208-210, 2009.

[5] V.V. Goryunova, O.V. Lukinova, T.I. Gorunova, "OntologicallyOriented methods of integration of modular ERP systems" [Proc. of the 2nd Russia and Pacific Conf. on Computer Technology and Applications]. Vladivostok, 2017.

[6] R. Wootton, The Development of Telemedicine. Century. Oxon: Radcliffe Medical Press, 2000, pp. 17-26.

[7] P.J. Heinzelmann, N.E. Lugne, J.K. Quedar, "Telemedicine in the future", J. of Telemedic. and Telecare, vol. 11, no. 8, pp. 384-390, 2005.

[8] P.A. Jennett et al., "The social and economic impact of telehealth: a systematic review", J. of Telemedic. and Telecare, vol. 9, no. 6, pp. 311-320, 2003.

[9] R. Swinfen, P. Swinfen, "Inexpensive telemedicine in developing countries", J. of Telemedic. and Telecare, 2002.

[10] T. Gavrilova, I. Leshcheva, Ontology Design and Individual Cognitive Peculiarities: A Pilot Study, Expert Systems with Applications. DOI: 10.1016/j.eswa.2015.01.008

[11] D.L. Brock, E.W. Schuster, S.J. Allen, P. Kar, "An Introduction to Semantic Modeling for Logistical Systems", J. of Busin. Logistics, vol. 26/2, 2009

[12] New Frontiers in Information and Production Systems Modeling and Analysis - Incentive Mechanisms, Competence Management, Knowledge-based Production, Series "Intelligent Systems Reference Library", Ed. by P. Rozewsky, D. Novikov, O. Zaikin, N. Bakhtadze. Berlin: Springer, 2016, 268 p.

[13] D. Novikov, Control Methodology. New York: Nova Sci. Publ., 2013, p. 76.

[14] V.V. Goryunova, "Modular ontological system technology in intelligent information systems", Information-measuring and operating systems, vol. 10,2010

[15] G.B. Kleiner, "System paradigm and system management”, Rus. Manag J., vol. 3, no. 6, pp. 27-50, 2001

[16] D.M. Gimémez, M. Vegetti, H.P. Leone, G.P. Henning, "Product ontology: Defining product-related concepts for logistics planning activities", Computers in Industry, vol. 59, no. 2-3, 2007.

[17] D.A. Shageev, T.A. Chukhontseva, "Universal express methodology for assessing and making managerial decisions of situational problems at the enterprise", Bull. of the Voronezh State Univer. of Engineer. Technol., vol. 81, no. 2, pp. 359-376, 2019. Retrieved from: https://doi.org/10.20914/2310-1202-2019-2-359-376

[18] G.S. Merzlikina, "The economic effectiveness of the "ecological health" of the region", Bull. of the Voronezh State Univer. of Engineer. Technol., vol 81, no 2, pp. 312-319, 2019. https://doi.org/10.20914/2310-1202-2019-2-312-319

[19] Yu.V. Zhuravlev, I.V. Kuksova, E.A. Hubertov, L.I. Churikov, "Assessment of the innovative development of the Russian Federation based on indicators of the concept and strategy of 2020", Bull. of the Voronezh State Univer. of Engineer. Technol., vol. 81, no. 2, pp. 377-382, 2019. Retrieved from: https://doi.org/10.20914/2310-1202-2019-2-377382 